\title{
INFLUENCE OF ROLLING FRICTION COEFFICIENT ON INTER-PARTICLE PERCOLATION IN A PACKED BED BY DISCRETE ELEMENT METHOD
}

Rolling friction representing the energy dissipation mechanism with the elastic deformation at the contact point could act directly on particle percolation. The present investigation intends to elucidate the influence of rolling friction coefficient on inter-particle percolation in a packed bed by discrete element method (DEM). The results show that the vertical velocity of percolating particles decreases with increasing the rolling friction coefficient. With the increase of rolling friction coefficient, the transverse dispersion coefficient decreases, but the longitudinal dispersion coefficient increases. Packing height has a limited effect on the transverse and longitudinal dispersion coefficient. In addition, with the increase of size ratio of bed particles to percolation ones, the percolation velocity increases. The transverse dispersion coefficient increases with the size ratio before $D / d<14$. And it would keep constant when the size ratio is greater than 14 . The longitudinal dispersion coefficient decreases when the size ratio increases up to $D / d=14$, then increases with the increase of the size ratio. External forces affect the percolation behaviours. Increasing the magnitude of the upward force (e.g. from a gas stream) reduces the percolation velocity, and decreases the dispersion coefficient.

Keywords: Inter-particle percolation, rolling friction coefficient, discrete element method, percolation velocity, dispersion coefficient

\section{Introduction}

COREX, a smelting reduction process, is regarded as a cost-efficient and environmental-friendly process, which produces hot metal from iron ore and coal [1-2]. It is a twostage process that involves pre-reduction in a shaft furnace, followed by final reduction and separation in a melter gasifier. Both shaft furnace and melter gasifier are typical countercurrent reactors, in which reducing gases flow upward through the packed bed while the charged solid particles flow downwards. In these flows, the motion of particles, as a densely packed bed, will have a significant impact on the flow of other phases, and thus on operation efficiency of the entire production. That is, the transient features of solids are crucial in the COREX process [3-10]. During burden distribution and particle descending processes, the mixing and segregation behaviour can be observed. One of the main reason is particle percolation. Fundamental studies of solid flow and segregation in COREX are very much based on particle percolation studies under different operating conditions. Besides, the chocking of shaft gas slots arises mainly from the insufficient percolation of dust particles in the gas and the fines in the burden. Thus, it is very necessary to make a fundamental study on the percolation phenomenon in a packed bed.

Inter-particle percolation may occur if two particles are of very different sizes. The smaller components drain through the larger ones simply due to the influence of gravity. This is normally termed as the spontaneous inter-particle percolation. Such percolation is a common phenomenon in nature and industries. A specific example arises in iron making blast furnace, where small sinter and/or pellet particles are loaded upon much larger coke particles, the smaller particles pass through the larger ones in descending motion under gravity [11]. The phenomenon of spontaneous inter-particle percolation has been investigated by means of various physical and numerical experiments in the past. Bridgwater and his colleagues [12-14] made a pioneering research in studying experimentally interparticle percolation, focusing on percolation velocity, residence time and radial distance distribution. Ippolito et al. [15] investigated experimentally the dispersion of small spherical beads moving under the effect of gravity inside a packing of large spheres. Richard et al. [16] used Monte Carlo method to analyze various properties relevant to percolation. Lomine and Oger $[17,18]$ performed experiments and discrete element method (DEM) simulation to analyze dispersion of particles through a porous structure. Rahman and Zhu $[19,20]$ and Li et al. [21] performed DEM to study the effect of particle properties on particle percolation behaviour in a packed bed. While these studies provide useful information, the effect of rolling friction on the inter-particle percolation is inadequate. Recently, Yu and Saxén [22] investigated the effect of DEM parameters including rolling friction on the inter-particle percolation of pellets into coke packing during burden descent in the blast furnace. However, the work has a lack of investigation in percolation behavious such as residence time distribution, longitudinal and transverse dispersion.

Actually, the material properties like size, density, friction and shape have great impact on particles flow and static

* SCHOOL OF MATERIALS AND METALLURGY, NORTHEASTERN UNIVERSITY, SHENYANG 110819, LIAONING, CHINA

\# Corresponding author: luozg@smm.neu.edu.cn 
characteristics. The rolling friction, specially, representing the energy dissipation mechanism with the elastic deformation at the contact point could act directly on particle dispersion. In DEM, it is also possible to consider the particle shape by selection of the rolling friction coefficient [23-25]. Therefore, a detailed study is necessary to clarify the influence of the rolling friction coefficient, which is related to the energy dissipation and shape factor, on the inter-particle percolation in a packed bed.

Based on the above background, this study focuses on the influence of the rolling friction on the spontaneous inter-particle percolation by means of DEM simulation. Percolation behaviours such as percolation velocity, residence time distribution, longitudinal and transverse dispersion are considered. The effects of the size ratio of packing particles to percolation ones, and external forces on the percolation behaviour are also considered.

\section{Theoretical Treatments}

\subsection{DEM}

Each single particle in a considered system undergoes both translational and rotational motion, described by Newton's 2nd law of motion. The forces and torques considered include those originating from the particle's contact with neighbouring particles, walls and surrounding fluids. The governing equations for translation and rotational motions of particle $i$ with $R_{i}$, mass $m_{i}$ and moment of inertia $I_{i}$ can be written as

$$
\begin{gathered}
m_{i} \mathrm{~d} \boldsymbol{v}_{i} / \mathrm{d} t=\sum_{j=1}^{k_{i}}\left(\boldsymbol{F}_{\mathrm{cn}, i j}+\boldsymbol{F}_{\mathrm{dn}, i j}+\boldsymbol{F}_{\mathrm{ct}, i j}+\boldsymbol{F}_{\mathrm{dt}, i j}\right)+m_{i} \boldsymbol{g} \\
\boldsymbol{I}_{i} \mathrm{~d} \boldsymbol{\omega}_{i} / \mathrm{d} t=\sum_{j=1}^{k_{i}}\left(\boldsymbol{T}_{i j}+\boldsymbol{M}_{i j}\right)
\end{gathered}
$$

where, $\boldsymbol{m}_{i}, \boldsymbol{I}_{i}, \boldsymbol{v} \mathrm{i}$, and $\boldsymbol{\omega}_{i}$ represent mass, rotational inertia, translational velocity, and rotational velocity of particle $i$, respectively; $\boldsymbol{F}_{\mathrm{cn}, i j}, \boldsymbol{F}_{\mathrm{ct}, i j}, \boldsymbol{F}_{\mathrm{dn}, i j}, \boldsymbol{F}_{\mathrm{dt}, i j}, \boldsymbol{T}_{i j}$, and $\boldsymbol{M}_{i j}$ represent normal and tangential contact force, normal and tangential damp force, and tangential and rolling friction torque of particle $j$ acting on particle $i$, respectively; $\boldsymbol{g}$ is gravitational acceleration; $k_{i}$ is the number of particles in contact with particle $i$; and $t$ is time. According to existing literatures [10,26-28], equations for contact force, damping force, friction force, and torque used here are listed in Table 1 , where, $R^{*}$ is the equivalent radius; $E^{*}$ is equivalent Young's modulus; $E$ is Young's modulus; $v$ is Possion's ratio; $\delta_{\mathrm{n}}$ is normal particle overlap; $\hat{\boldsymbol{n}}$ is a unit vector from the center of the particle to the contact point; $m_{i j}$ is equivalent mass; $\boldsymbol{v}_{\mathrm{n}, i j}$ is the normal relative velocity of particle $i$ and $j ; \mu_{s}$ is sliding frictional coefficient; $\delta_{t, i j}$ is the particle tangential overlap; $\delta_{\mathrm{t}, i j}$, max is the maximum particle tangential overlap; $\hat{\boldsymbol{\delta}}_{\mathrm{t}}$ is the unit vector of particle tangential overlap; $\boldsymbol{v}_{\mathrm{t}, i j}$ is the tangential relative velocity of particle $i$ and $j ; \boldsymbol{R}_{i j}$ is the vector from the mass center of particle $i$ to particle $j ; \mu_{\mathrm{r}, i j}$ is rolling friction coefficient; $\hat{\boldsymbol{\omega}}_{\mathrm{t}, j j}$ is the unit vector of particle angular velocity.

\subsection{Simulation conditions}

The simulation setup, as shown in Fig. 1, is made of a cylindrical container of $\phi 15 D \times 15 D$ filled with a packing of monosize large particles (which are referred to as packing particles here) of diameter $D$. This packing is built by random gravity deposition of the particles. This procedure gives a reproducible porosity around 0.4 . Small particles (percolating particles) of diameter $d$ are put on the top of the packed bed. They are generated randomly at the centerline of the column in a circle of diameter of $1 D$. These percolating particles pass through the packed bed towards the bottom of the column under gravity. Their dynamic details are recorded for analysis. The parameters used in the present simulations are listed in Table 2. In this work, the same simulation process is repeated three times and each packing is rebuilt for each simulation.

\begin{tabular}{|c|c|c|c|}
\hline \multicolumn{2}{|c|}{ Force and torque } & Symbol & Equation \\
\hline \multirow{2}{*}{ Normal } & Contact force & $\boldsymbol{F}_{\mathrm{cn}, i j}$ & $-4 / 3 E^{*} \sqrt{R^{*}} \delta_{\mathrm{n}}^{3 / 2} \hat{\boldsymbol{n}}$ \\
\hline & Damping force & $\boldsymbol{F}_{\mathrm{dn}, i j}$ & $-\eta_{\mathrm{n}}\left(6 m_{i j} E^{*} \sqrt{R^{*} \delta_{n}}\right)^{1 / 2} \boldsymbol{v}_{\mathrm{n}, i j}$ \\
\hline \multirow{2}{*}{ Tangential } & Contact force & $\boldsymbol{F}_{\mathrm{ct}, i j}$ & $-\mu_{\mathrm{s}}\left|\boldsymbol{F}_{\mathrm{c}, i j}\right|\left(1-\left(1-\delta_{\mathrm{t}, j} / \delta_{\mathrm{t}, i j, \max }\right)^{3 / 2}\right) \hat{\boldsymbol{\delta}}_{\mathrm{t}} \quad\left(\delta_{\mathrm{t}, j}<\delta_{\mathrm{t}, j, \max }\right)$ \\
\hline & Damping force & $\boldsymbol{F}_{\mathrm{dt}, i j}$ & $-\eta_{\mathrm{s}}\left(6 \mu_{\mathrm{s}} m_{i j}\left|\boldsymbol{F}_{\mathrm{ch}, i j}\right| \sqrt{1-\delta_{\mathrm{t}, j} / \delta_{\mathrm{t}, i, \max }} / \delta_{\mathrm{t}, i, \mathrm{max}}\right)^{1 / 2} \boldsymbol{v}_{\mathrm{t}, i j} \quad\left(\delta_{\mathrm{t}, i j}<\delta_{t, i, \max }\right)$ \\
\hline \multicolumn{2}{|c|}{ Friction force } & $\boldsymbol{F}_{\mathrm{t}, i j}$ & $-\mu_{\mathrm{s}}\left|\boldsymbol{F}_{\mathrm{cn}, i j}\right| \hat{\boldsymbol{\delta}}_{\mathrm{t}} \quad\left(\delta_{\mathrm{t}, j}>\delta_{\mathrm{t}, j, \mathrm{max}}\right)$ \\
\hline \multicolumn{2}{|c|}{ Gravity } & $\boldsymbol{F}_{\mathrm{g}, i}$ & $m_{i} \boldsymbol{g}$ \\
\hline \multicolumn{2}{|c|}{ Tangential torque } & $T_{i j}$ & $\boldsymbol{R}_{i j} \times\left(\boldsymbol{F}_{\mathrm{ct}, i j}+\boldsymbol{F}_{\mathrm{dt}, i j}\right)$ \\
\hline \multicolumn{2}{|c|}{ Rolling friction torque } & $\boldsymbol{M}_{i j}$ & $\mu_{\mathrm{r}, j, j}\left|\boldsymbol{F}_{\mathrm{cn}, i j}\right| \hat{\omega}_{\mathrm{t}, i j}^{n}$ \\
\hline
\end{tabular}

Components of forces and torques acting on particle $i$

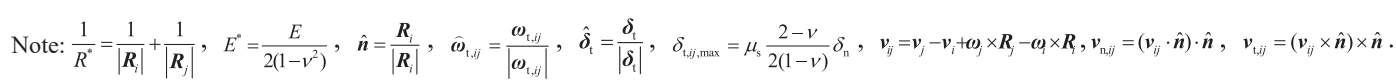


Each numerical data which are presented in this paper are coming from a statistical mean of several simulations. The error bars are deduced from these replicate simulations.

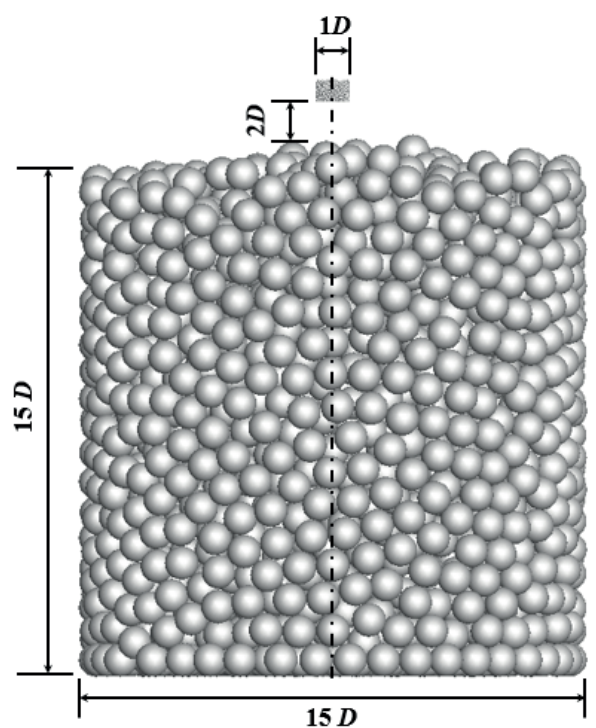

Fig. 1. Geometry of the model used in this work

Particle properties and simulation conditions

\begin{tabular}{|c|c|}
\hline \hline Variables & Value \\
\hline Diameter of packed particle, $D(\mathrm{~m})$ & 0.01 \\
\hline Percolating particle diameter, $d$ & $0.02-0.1 D$ \\
\hline Percolating particle number, $N$ & 500 \\
\hline Sliding frictional coefficient, $\mu_{\mathrm{s}}$ & 0.3 \\
\hline Rolling frictional coefficient, $\mu_{\mathrm{r}}$ & $0.001-0.1 D$ \\
\hline Young's modulus, $E(\mathrm{~Pa})$ & $50000 \mathrm{~g} \pi \mathrm{D} \rho / 6$ \\
\hline Poisson's ratio, $v_{\mathrm{p}}$ & 0.3 \\
\hline Damping coefficient, $c$ & 0.3 \\
\hline Time step, $\Delta t(\mathrm{~s})$ & $1.0 \times 10^{-7}$ \\
\hline
\end{tabular}

TABLE 2

\section{Results and discussion}

\subsection{Model validity}

In this work, the present DEM model is validated by the angle of repose of coarse spheres. The simulations were carried out under conditions similar to those used in previous physical experiments [29]. The so-called discharging method is used to examine the angle of repose. The physical experiments were carried out in a rectangular container with a fixed middle plate and two side outlets. Particles with diameter of $2 \mathrm{~mm}$ (particle density $=2500 \mathrm{~kg} / \mathrm{m}^{3}, \mu_{\mathrm{r}}=0.05 \mathrm{~mm}$ and $\mu_{\mathrm{s}}=0.4$ ) were employed. Fig 2 shows the typical sandpiles constructed by physical experiments and numerical simulations with different container thicknesses. Because of the relatively small number of particles, the numerical simulation sometimes does not produce a sandpile of smooth straight surface. Nevertheless, the results clearly indicated that the angle of repose decreases with increasing container thickness, and the numerical simulations and physical experiments are quite comparable. Therefore, the present model is suitable to carry out further simulation.

\subsection{Percolation behaviour}

The main phenomena of the spontaneous inter-particle percolation are the longitudinal and transverse dispersions. The longitudinal direction is referred to the flow direction, and the transverse one is referred to the direction perpendicular to the flow direction. The percolation velocity reflects, to a degree, the dispersion property of percolating particles. In the following, the analysis will focus on the percolation velocity, lateral and transverse dispersion behaviour in a packing bed.

Fig. 3 presents the evolution of normalized mean vertical velocity as a function of normalized time for $D / d=10$ and $\mu_{\mathrm{r}}=0.001 D$. The time is set to be proportional to the free fall time to pass a single large sphere diameter. The velocity is
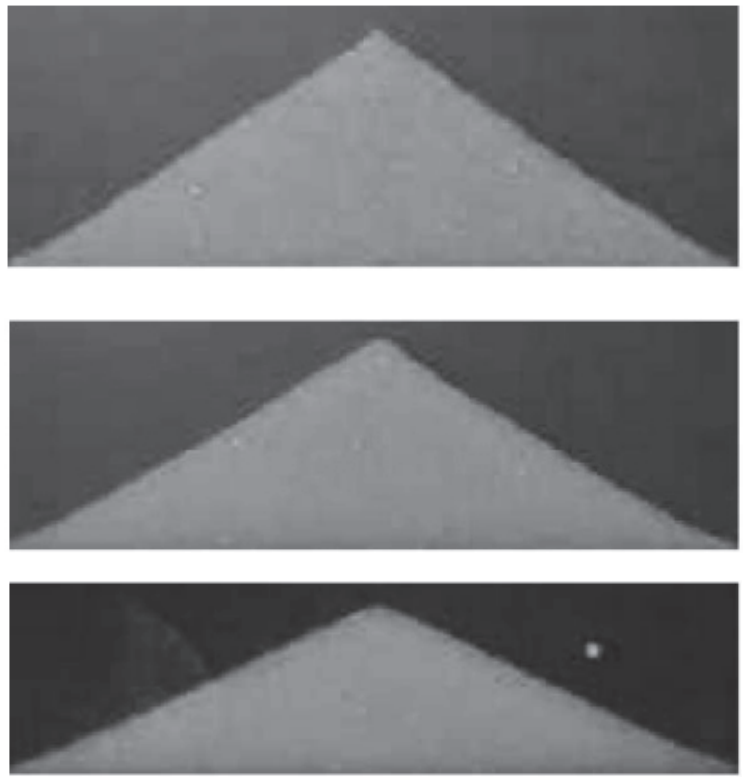

(a)

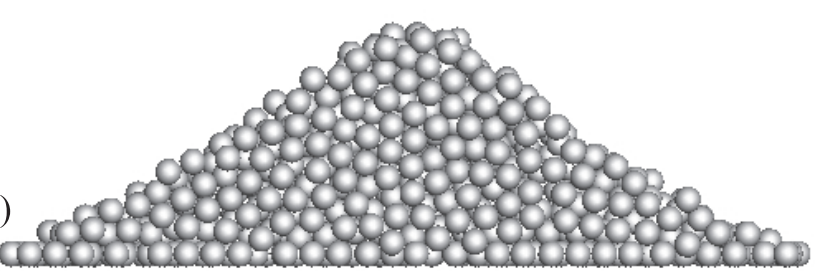

(b)

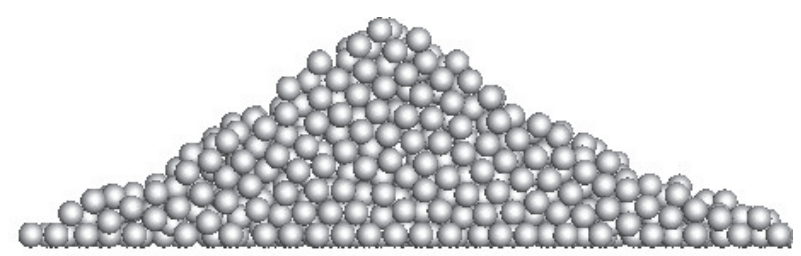

(c)

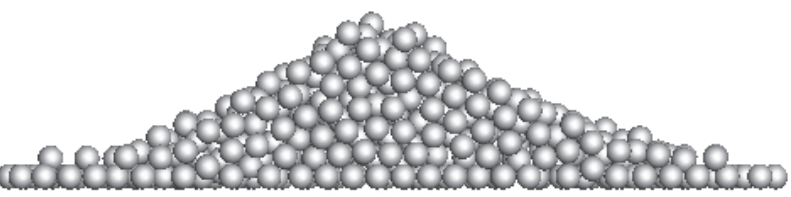

Fig. 2. Sandpiles generated via physical and numerical with different container thickness: (a) $w=4 D$; (b) $w=12 D$; (c) $w=20 D$ 
expressed in units of free fall velocity reached after falling over one large particle. It can be seen form Fig.3, at the very beginning, the mean vertical velocity increases rapidly due to the free fall of small particles. After a short time of free fall, the

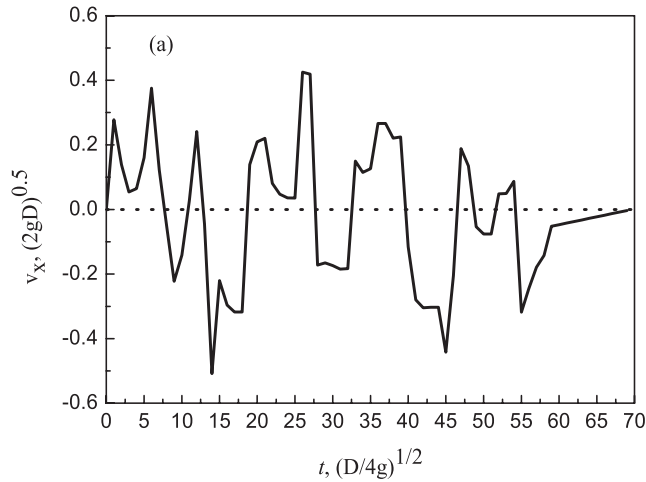

mean velocity decreases progressively towards a steady value. This phenomenon can be related to previous results: for the case of particles falling down in a random packed bed of larger particles, the vertical velocity is a constant $[12,13,17,19]$.

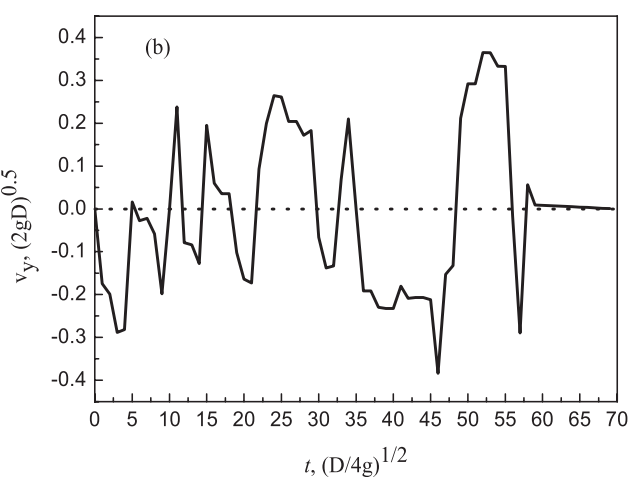

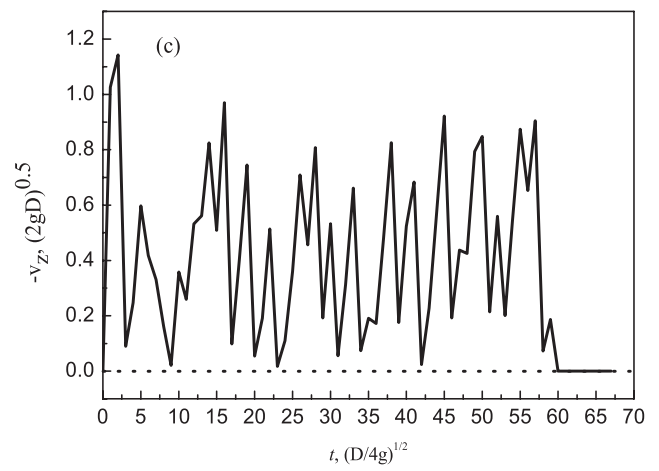

Fig. 4. Evolution of the velocity of a representative percolating particle during the percolation process when $D / d=10, \mu_{\mathrm{r}}=0.001 D$ and $H=15 \mathrm{D}$ : (a) $x$-direction, (b) $y$-direction, (c) $z$-direction
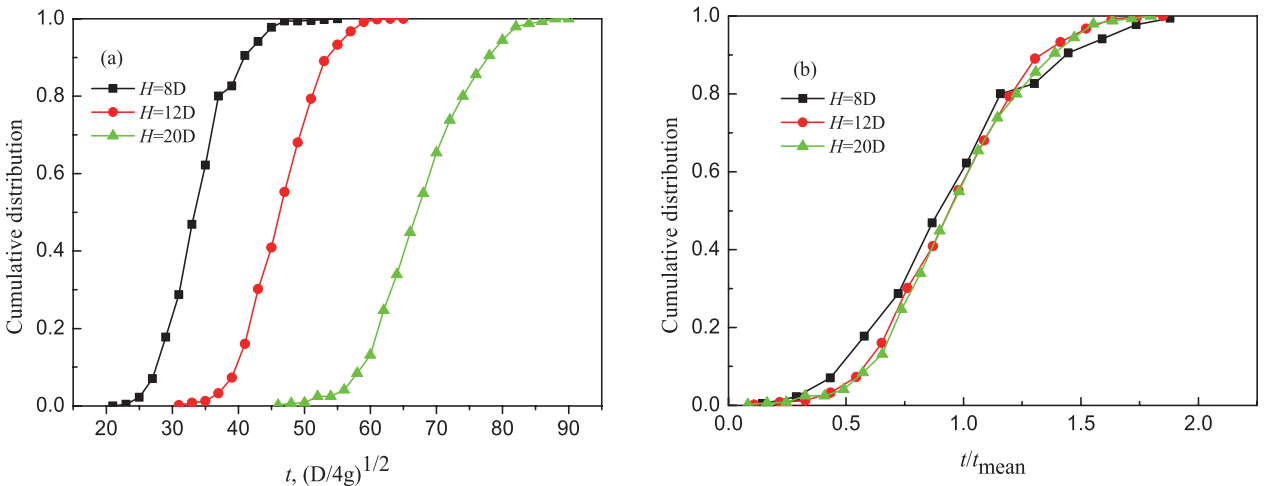

Fig. 5. Cumulative distributions of: (a) residence time, and (b) normalized residence time, $t / t_{\text {mean }}\left(t_{\text {mean }}\right.$ is the average of the residence time) for different packing heights when $D / d=10$ and $\mu_{\mathrm{r}}=0.001 D$
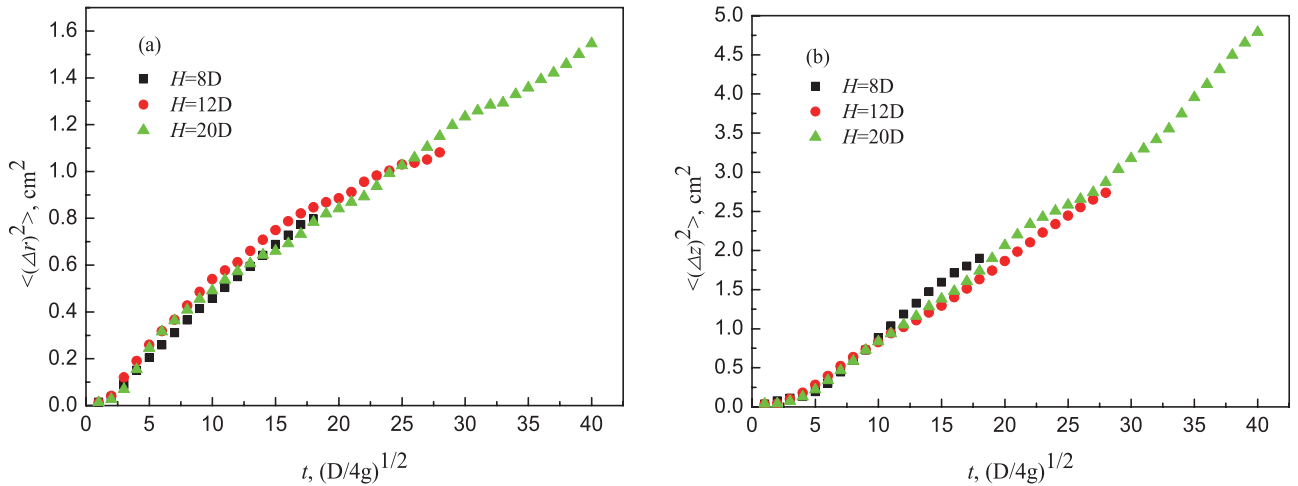

Fig. 6. Time evolutions of (a) $\left\langle(\Delta r)^{2}\right\rangle$, and (b) $\left\langle(\Delta z)^{2}\right\rangle$ for $D / d=10$ and $\mu_{\mathrm{r}}=0.001 D$ 
Although the mean vertical velocity of percolating particles is constant, the variation of the velocity with time is very complex. Fig 4 shows the evolution of the velocity of a representative percolating particle during the percolation process of $D /$ $d=10$ and $\mu_{\mathrm{r}}=0.001 D$. It can be observed that the magnitudes of percolation velocity fluctuate largely and irregularly. The fluctuation of horizontal velocities ( $x$-direction and $y$-direction) could reflect the stochastic motion of the percolating particle. Such stochastic motion could lead to dispersion of percolating particle within a region in the packed bed. The fluctuation of the vertical velocity is caused by the impact between the percolating and packing particles. When the percolating particles do not meet packing particles, their velocities increase rapidly. The collision of the percolating particles with packing particles resists the downward movement of the percolating particle and reduces its vertical velocity.

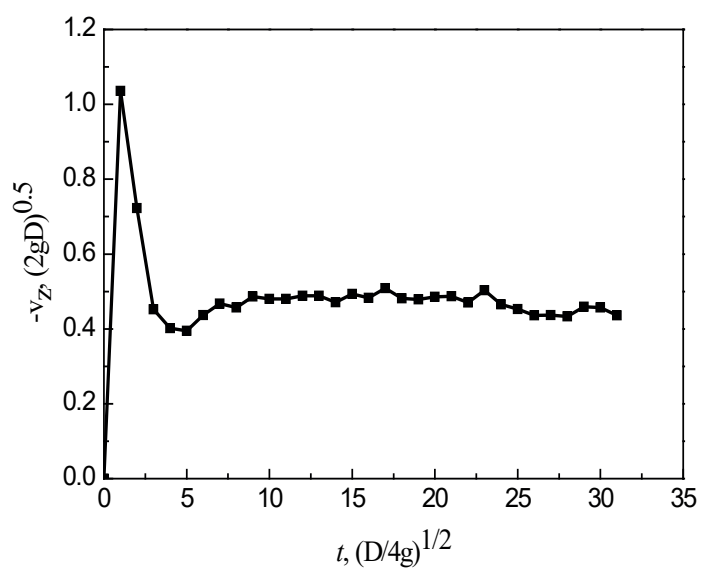

Fig. 3. Evolution of mean vertical velocity during the percolation process when $\mathrm{D} / \mathrm{d}=10, \mathrm{~N}=500$ and $\mu \mathrm{r}=0.001 \mathrm{D}$

Fig. 5 shows the cumulative distributions of residence time of percolating particles at different bed heights for $D / d=10$ and $\mu_{\mathrm{r}}=0.001 D$. It is obvious that particles take different times to reach the bottom of the packing bed under different heights. The higher the packing bed is, the longer is the residence time. However, it is meaningful to consider if the cumulative time distribution is dependent on the packing height, when plotted against dimensionless time $t / t_{\text {mean }}$. It can be seen from Fig. 5(b) that the bed height has limited effect on the normalized cumulative residence time distributions, and this does imply that the residence time increases proportionally with bed height. The more important result is that this is another evidence indicating that the percolation velocity of particles is a constant.

The dispersion of percolating particles is a random walk process. The DEM model is possible to access individual particle positions, anywhere at any time, inside the packing of larger spheres. The position of particle $k$ in the horizontal plane can be described as $r_{k}^{2}=x_{k}^{2}+y_{k}^{2}$, where $x_{k}$ and $y_{k}$ are the particle positions. So the variance of the position distributions of the $N$ moving particles in this plane is

$$
\left\langle(\Delta r)^{2}\right\rangle=\frac{1}{N} \sum_{k=1}^{N}\left(r_{k}-\langle r\rangle\right)^{2}
$$

where $\langle r\rangle=\frac{1}{N} \sum_{k=1}^{N} r_{k}$ In the same manner, if the particle position in the flow direction is denoted by $z_{k}$, it can be written as

$$
\left\langle(\Delta z)^{2}\right\rangle=\frac{1}{N} \sum_{k=1}^{N}\left(z_{k}-\langle z\rangle\right)^{2}
$$

where, $\langle z\rangle=\frac{1}{N} \sum_{k=1}^{N} z_{k}$.

Then, the transverse and axial dispersion coefficients $D_{\perp}$ and $\mathrm{D} \|$ can be defined from the time evolution of $\left\langle(\Delta r)^{2}\right\rangle$ and $\left\langle(\Delta z)^{2}\right\rangle$ with Einstein-Smoluchowski equation as follows

$$
\left\langle(\Delta r)^{2}\right\rangle=2 D_{\perp} t \text { and }\left\langle(\Delta z)^{2}\right\rangle=2 D_{\|} t
$$

Fig. 6 presents the variances, $\left\langle(\Delta r)^{2}\right\rangle$ and $\left\langle(\Delta z)^{2}\right\rangle$ calculated with Eqs. (3) and (4), of particle position distribution for different packing bed heights versus time. It can be seen that the relationship between the dispersion position and flow time is linear. The linear evolution of the two variances with time is a typical feature of a diffusive property. The diffusive motion of a blob of small particles is also confirmed by the previous work [18]. Furthermore, as expected, the packing bed height has little effect on the percolating particle position. As the height has limited effect on the normalized cumulative residence time and dispersion coefficient, the description of the percolation behaviour can be simplified by fixing the packing height $H=15 D$ in later work.

\subsection{Effect of rolling friction coefficient}

Rolling friction provides an effective mechanism to control the translational and rotational motions and largely determine the energy dissipation at the contact point. It can also be considered as a shape factor in DEM model [23-25]. To quantify the effect of rolling friction coefficient on the inter-particle percolation in a packed bed, the percolation velocity, residence time, longitudinal and transverse dispersions are discussed here. The effect of rolling friction coefficient on percolation velocity is shown in Fig. 7. It can be observed that, for higher rolling friction coefficient, the percolation velocity is lower. When percolating particles come into contact with packing particles, they would move downwards and experience multiple collisions. All the contact between the percolating and packing particles would result in a rolling resistance due to elastic hysteresis losses or viscous dissipation. Therefore, larger rolling friction coefficient would lead to smaller percolation velocity.

Fig. 8 shows the statistic distributions of residence time for different rolling fraction coefficients when $D /$ $d=10, N=500$. Bridgwater [13] experimentally studied the residence time distribution of percolating particles and showed that the residence time distribution is roughly similar to a normal distribution except for the lead particles. Similar trend can also be observed in the present simulation when the rolling fraction coefficient $\mu_{\mathrm{r}}=0.001 D$. However, with large rolling fraction, the distribution curve shifts to the right, becomes wider, and does not show a normal distribution. This is because that the higher the rolling friction coefficient is, the larger is the energy dissipation, 


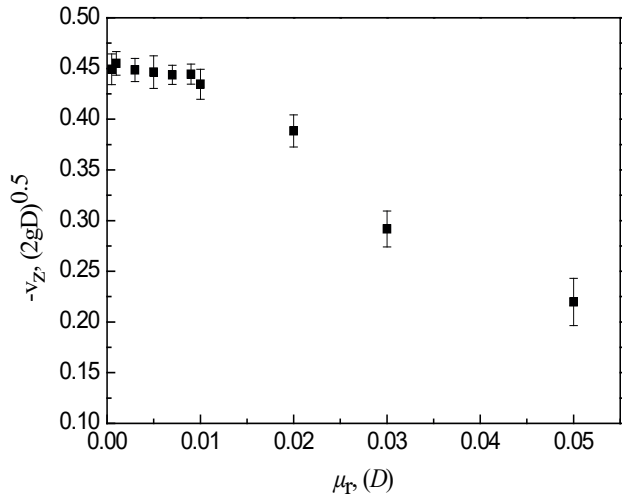

Fig. 7. Percolation velocity with different rolling friction coefficients for $D / d=10$

a)

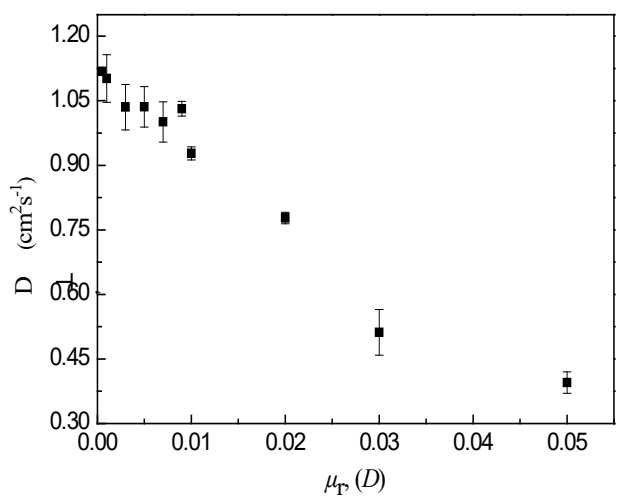

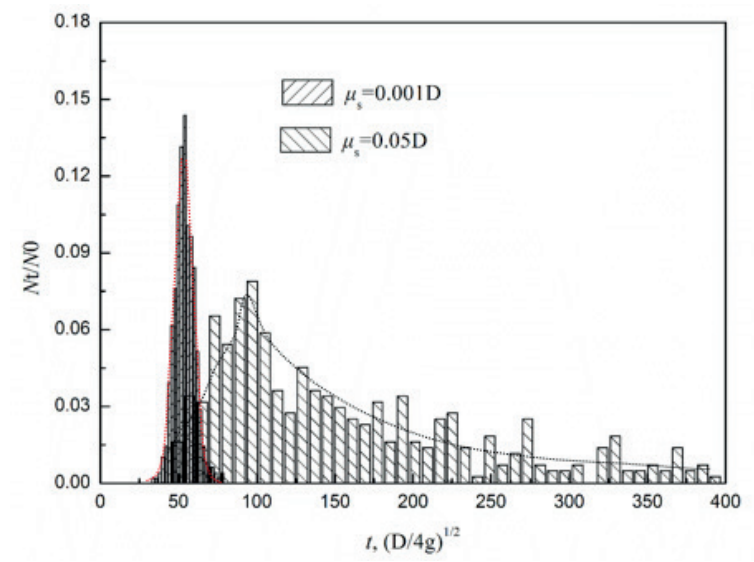

Fig. 8.. Statistic distributions of residence time with different rolling fraction coefficients for $D / d=10$

b)

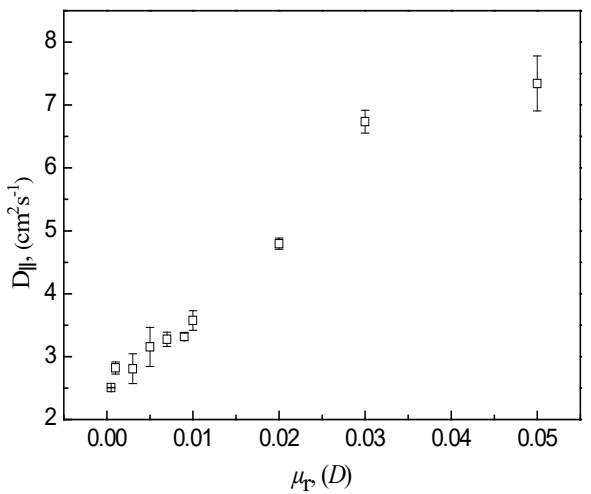

Fig. 9. Variations of (a) transverse dispersion and (b) longitudinal dispersion coefficient with $\mu \mathrm{r}$ for $D / d=10$

and then the time for the particles to reach the bottom of the packed bed will be longer.

The variations of the transverse dispersion coefficient and longitudinal dispersion coefficient with $\mu_{\mathrm{r}}$ are shown in Fig. 9. From the Fig. 9 (a), it can be seen that the larger the rolling friction coefficient is, the less is the particles transverse dispersion. On the other hand, Fig. 9 (b) shows that, the longitudinal dispersion $D \|$ increases with the increasing of the rolling friction coefficient. The transverse dispersion coefficient varies in opposition to the longitudinal dispersion. For smaller rolling friction coefficient, the energy dissipation is reduced and the particles can explore laterally the porous medium more easily due to the more chance to bounce around the packing particles. Yu and Saxén also found that a low rolling friction between pellets promotes the percolation [22]. On the other side, when the rolling friction coefficient is increased, more relative kinetic energy can be dissipated, and the gravity force is more important. Hence, longitudinal crossing of individual pore is easier and leads to an increase in the longitudinal dispersion coefficient.

\subsection{Effect of size ratio $D / d$}

The variation of percolation velocity with particle size for different rolling friction coefficients is confirmed in Fig.
10. It can be observed that, with increasing the particle size ratio, the percolation velocity increases. This can be easily explained by considering a single particle falling down toward a pore. The particle trajectory must be aligned with the pore hole to pass through it without bouncing around for big ratio of particle size. If not, the smaller the size ratio is, the more important is the collisions of particles. In such a case, the downward motion of the percolating particle is more difficult and leads to a decrease in the percolation velocity.

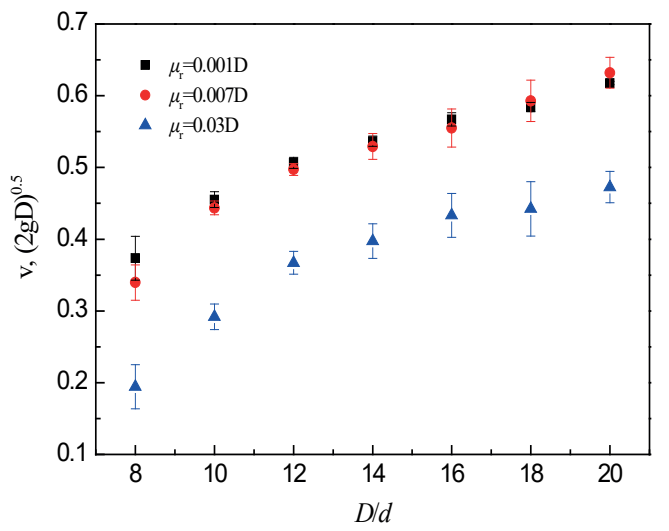

Fig. 10. Variation of percolation velocity with particle size ratio for different rolling friction coefficients 

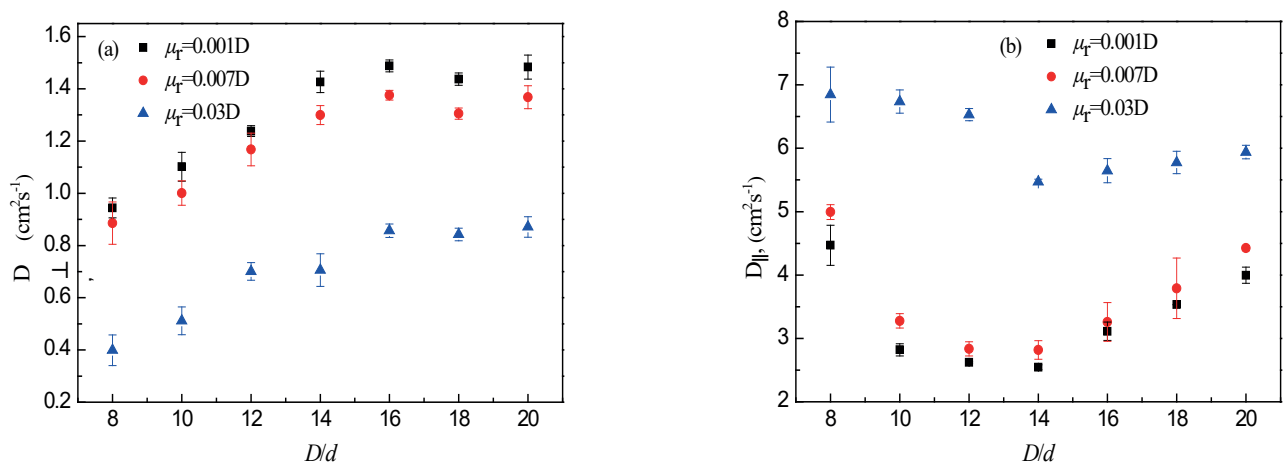

Fig. 11 Variations of (a) transverse dispersion and (b) longitudinal dispersion coefficients with $D / d$

Fig. 11 shows the variations of transverse dispersion and longitudinal dispersion coefficients with the particle size ratio. Fig. 11 (a) demonstrates that, when the size ratio is smaller than 14 , the transverse dispersion coefficient increases with the size ratio. The smaller the particles are, the longer distance they can laterally move. When the size ratio is greater than 14 , it can be seen that the transverse dispersion coefficient is nearly constant. The main reason can be summarized as: when $D / d$ is small, the pore throats acting like gates in packed bed would create a "gate or valve effect" which could lead to an impedance on the transverse motion of percolating particle, hence reduces the transverse dispersion. When $D / d$ is greater than 14 , the size of percolating particles is smaller compared with the pore throats and the "gate or valve" effect on transverse motion will no longer function. So the transverse dispersion coefficient stays constant when the size ratio $D / d>14$. The similar tendency is also observed in a previous work by Lomine and Oger [18]. Fig. 11 (b) shows that, the longitudinal dispersion coefficient decreases when the size ratio increases up to $D / d=14$, then increases with further increase of the size ratio. The phenomena can be explained as: when $D / d$ is small, the particles have a smaller probability of moving outside a pore in the horizontal direction and thus a greater probability of passing through the vertical pore. On the other hand, when $D / d$ is greater than 14 , the transverse diffusive motion is almost the same, but the particle with smaller diameter could have more chance to descend directly in the flow channel without contacting with the packed particle. Therefore, the longitudinal dispersion coefficient increases with the increase of size ratio when $D / d>14$.

\subsection{Effect of external force}

A countercurrent gas flow is the typical practice in a shaft furnace. In such cases, the particles are subjected to an external force. Such a force (upward direction) will contribute to the movement of small particles, and hence influence the particle percolation velocity. In reality, the gas drag force on each particle is depended on the gas flow distribution corresponding to the local permeability. However, for brevity, it is assumed that the particles are only affected by a constant force in upward direction, $f_{\mathrm{e}}$, in addition to contact forces and gravity. The effect of this external force on the particle percolation velocity is shown in Fig. 12. It can be seen that increasing the magnitude of the force in upward direction reduces the particle velocity. Further, the percolation velocity and the force are found to have an approximate linear relationship. The similar phenomenon is also found in a previous work [20].
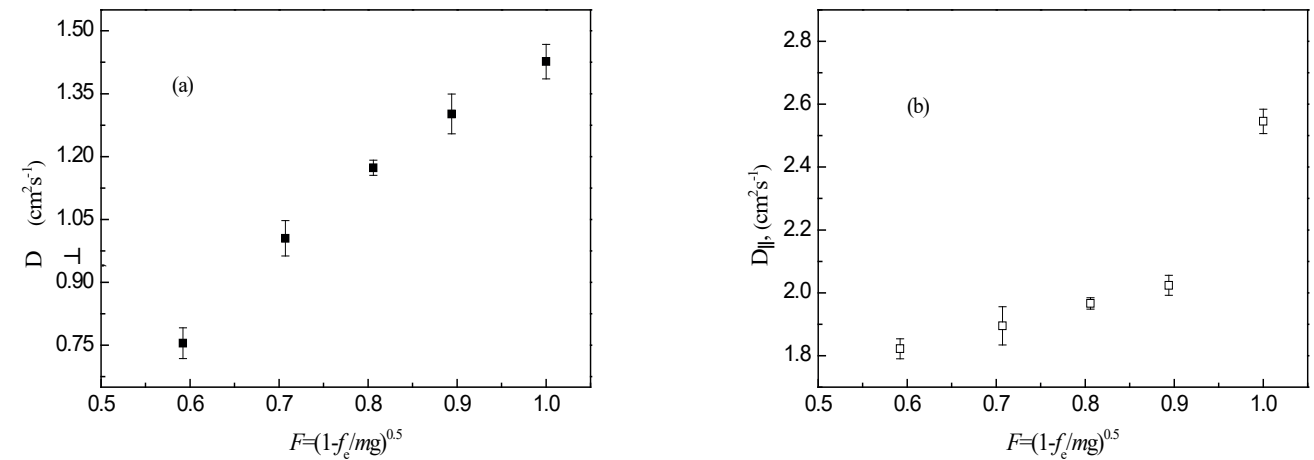

Fig. 13. Variations of (a) transverse dispersion and (b) longitudinal dispersion coefficients with upward external force 


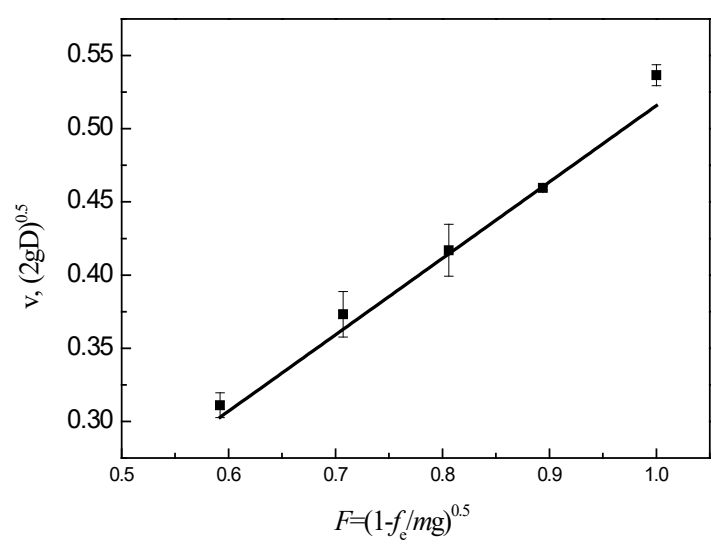

Fig. 12. Relationship between dimensionless percolation velocity and external force for $D / d=14$ and $\mu_{\mathrm{r}}=0.001 D$

Fig. 13 shows the variations of transverse dispersion and longitudinal dispersion coefficients with the external force. It can be observed that both of the transverse and longitudinal dispersion increase with the decreasing of the external force. For smaller external force, the vertical acceleration is larger and the particle kinetic energy is also higher. Percolating particles with higher energy can disperse explore laterally the packing bed more easily. At the same time, the larger vertical acceleration could enhance the dispersion in the vertical direction.

\section{Conclusions}

The influence of rolling friction on the spontaneous inter-particle percolation phenomenon has been studied by means of DEM simulation. Percolation behavious such as percolation velocity, residence time distribution, longitudinal and transverse dispersion have been examined. The following results are obtained.

1. The vertical velocity of percolating particles moving down through a random packed bed of larger particles is a constant, but it decreases with increasing rolling friction coefficient. The residence time distribution curve becomes wider and does not follow the normal distribution for the case with high rolling friction coefficient.

2. The rolling friction coefficient affects the dispersion behaviour of percolating particles. With the increase of rolling friction coefficient, the transverse dispersion coefficient decrease, but the longitudinal dispersion coefficient increase.

3. The effect of packing height on the residence time can be eliminated in the analysis of the residence time when they are normalized by the average residence time. Packing height has limited effect on the transverse and longitudinal dispersion coefficient.

4. With the increase of size ratio of packing particles to percolating ones, the percolation velocity increases. The transverse dispersion coefficient increases with the size ratio before $D / d<14$, and keeps constant when size ratio is higher than 14. The longitudinal dispersion coefficient decreases when the size ratio increases up to the ratio $D / d=14$, then increases when size ratio is larger.
5. The external force affects the percolation behavious. Increasing the magnitude of an upward force reduces the percolation velocity, and decreases the dispersion coefficient.

\section{Acknowledgments}

The authors would like to thank National Key Technology R\&D Program in "12th Five-Year Plan" of China (Grant No. 2011BAE04B02) and National Natural Science Foundation of China (Grant No. 51174053) for their financial support.

\section{REFERENCES}

[1] B. Anameric, S.K. Kawatra, Miner. Process. Extr. Metall. Rev. 30, 1-51 (2008).

[2] Y.X. Qu, Z.S. Zou, Y.P. Xiao, ISIJ Int. 52, 2186-2193 (2012).

[3] M.Y. Kou, S.L. Wu, W. Shen, K.P. Du, L.H. Zhang, J. Sun, ISIJ Int. 53, 2080-2089 (2013).

[4] Q.F. Hou, M. Samman, J. Li, A.B. Yu, ISIJ Int. 54, 1772-1780 (2014).

[5] H. Zhou, Z.S. Zou, Z.G. Luo, T. Zhang, Y. You, H.F. Li, Ironmaking Steelmaking 42, 209-216 (2015).

[6] Q.F. Hou, J. Li, A.B. Yu, Steel Res. Int. 86, 626-635 (2015).

[7] M.Y. Kou, S.L. Wu, G. Wang, B.J. Zhao, Q.W. Cai, Steel Res. Int. 86, 686-694 (2015).

[8] H. Zhou, Z.G. Luo, Z.S. Zou, T. Zhang, Y You, Steel Res. Int. 86, 1073-1081 (2015).

[9] H. Zhou, Z.G. Luo, T. Zhang, Y. You, Z.S. Zou, Ironmaking Steelmaking 42, 774-784 (2015).

[10] H. Zhou, Z.G. Luo, T. Zhang, Y. You, Z.S. Zou, Y. S. Shen, ISIJ Int. 56, 245-254 (2016).

[11] Y.W. Yu, A. Westerlund, T. Paananen, H. Saxén, ISIJ Int. 51, 1050-1056 (2011).

[12] J. Bridgwater, N.W. Sharpe, D.C. Stocker, Trans. Inst. Chem. Eng. 47, 144-199 (1969).

[13] J. Bridgwater, N.D. Ingram, Trans. Inst. Chem. Eng. 49, $163-$ 169 (1971).

[14] A.M. Scott, J. Bridgwater, Powder Tech. 14, 177-183 (1976).

[15] I. Ippolito, L. Samson, S. Bourles, J.P. Hulin, Eur. Phys. J. E 3, 227-236 (2000).

[16] P. Richard, L. Oger, J. Lemaître, L. Samson, N.N. Medvedev, Granular Matter 1, 203-211 (1999).

[17] F. Lomine, L. Oger, J. Stat. Mech.: Theory Exp. July, P07019 (2006).

[18] F. Lomine, L. Oger, Phys. Rev. E 79, 051307 (2009).

[19] M. Rahman, H. P. Zhu, A. B. Yu, J. Bridgwater, Particuology 6, 475-482 (2008).

[20] H. P. Zhu, M. Rahman, A. B. Yu, J. Bridgwater, P. Zulli, Miner. Eng. 22, 961-969 (2009).

[21] J. Li, A.B. Yu, J. Bridgwater, S. L. Rough, Powder Tech. 203 , 397-403 (2010).

[22] Y.W. Yu, H. Saxén, ISIJ Int. 52, 788-796 (2012).

[23] S. Natsui, S. Ueda, M. Oikawa, Z.Y. Zheng, J. Kano, R. Inoue, T. Ariyama, ISIJ Int. 49, 1308-1315 (2009).

[24] Q. Li, M.X. Feng, Z. S. Zou, ISIJ Int. 53, 1365-1371 (2013).

[25] T. Ariyama, S. Natsui, T. Kon, S. Ueda, S. Kikuchi, H. Nogami, ISIJ Int. 54, 1457-1471 (2014). 
[26] H.F. Li, Z.G. Luo, Z.S. Zou, J.J. Sun, L.H. Han, Z.X. Di, J. Iron Steel Res. Int. 19, 36-42 (2012).

[27] J.J. Sun, Z G. Luo, Z.S. Zou, Powder Technol. 281, 159-166 (2015).
[28] L.H. Han, Z.G. Luo, H. Zhou, Z.S. Zou, Y.Z. Zhang, J. Iron Steel Res. Int. 22, 304-310 (2015).

[29] Y.C. Zhou, B.H. Xu, A.B. Yu, P. Zulli, Powder Tech. 125, 4554 (2002). 
